\title{
Prevalencia de anemia en gestantes, Hospital Regional de Pucallpa, Perú
}

\author{
César Becerra, ${ }^{1}$ Gustavo F. Gonzales, ${ }^{2}$ Arturo Villena, ${ }^{2}$ \\ Doris de la Cruz ${ }^{2}$ y Ana Florián ${ }^{1}$
}

RESUMEN Las encuestas demográficas de salud y población del Perú indican que las tasas globales de fecundidad, la proporción de adolescentes embarazadas y la mortalidad maternoinfantil son más altas en la selva que en otras zonas del país. Las parasitosis intestinales endémicas agravan el riesgo de anemia ya generalmente presente en las embarazadas por deficiencias de hierro, ácido fólico y otros nutrientes. En muchos países latinoamericanos, esa es la complicación más frecuente del embarazo y está asociada con partos pretérmino, bajo peso al nacer y mortalidad perinatal. Los estudios realizados sobre este tema en la selva peruana son escasos y no se dispone de estimaciones confiables de la prevalencia de anemia durante la gestación. Los autores se propusieron determinar la prevalencia de anemia en mujeres gestantes que acudian al Hospital Regional de Pucallpa, en la selva del Perú, entre enero de 1993 y junio de 1995. El estudio de corte transversal se basó en los registros de control prenatal y de parto de 1015 embarazadas y permitió estudiar la asociación entre la prevalencia de anemia y variables como edad cronológica, escolaridad, número de gestaciones previas y peso de la madre al inicio del embarazo. También se compararon los valores de la hemoglobina materna con el peso de los recién nacidos.

La prevalencia de anemia en la población de gestantes fue de 70,1\%, valor que no se modificó por efecto de la edad materna, la escolaridad ni el intervalo intergenésico. La prevalencia de anemia se asoció directamente con el número de gestaciones e inversamente con la ganancia de peso durante el embarazo. La tasa de mortalidad perinatal fue de 37,7 por 1000 nacidos. Ni esta tasa ni el peso de los recién nacidos resultaron asociados con el grado de anemia de la madre. El análisis de regresión multivariado muestra que el peso de la madre al inicio de la gestación $(\mathrm{P}=0,0001)$, el peso ganado durante la gestación $(\mathrm{P}=0,0001)$ y el número de gestaciones $(\mathrm{P}=0,008)$ predicen el peso del recién nacido. Los resultados indican que la alta prevalencia de anemia en las gestantes de Pucallpa no se asocia con un bajo peso al nacer ni con una alta mortalidad perinatal. En estudios futuros deben investigarse las causas principales de la anemia que padecen las embarazadas de Pucallpa y sus efectos en el desarrollo psicomotor de sus hijos.

En los países en desarrollo, las tasas globales de fecundidad, mortalidad

\footnotetext{
1 Hospital Regional de Pucallpa, Departamento de Ginecología y Obstetricia, Pucallpa, Perú.

2 Universidad Peruana Cayetano Heredia, Departamento de Ciencias Fisiológicas e Instituto de Investigaciones de la Altura, Lima, Perú. La correspondencia y las solicitudes de separatas deben dirigirse a Gustavo F. Gonzales a la siguiente dirección postal: Universidad Peruana Cayetano Heredia, Instituto de Investigaciones de la Altura, Apartado postal 1843, Lima, Perú.
}

infantil y mortalidad materna son elevadas. Asimismo, la prevalencia de malnutrición infantil es alta y a menudo se traduce en retraso del crecimiento y del desarrollo y en enfermedades de origen alimentario, tales como la anemia (1).

En el Perú, la población muestra un crecimiento acelerado y la tasa de mortalidad maternoinfantil es alta. La situación representada por estos indi- cadores se torna más patente y alarmante cuando su análisis se estratifica por zonas geográficas. Según los resultados de la Encuesta Demográfica y de Salud Familiar, la tasa global de fecundidad es más alta en la selva que en otras zonas del país, al igual que la proporción de adolescentes embarazadas y la mortalidad maternoinfantil. En cambio, la proporción de niños escolarizados es menor en la selva (2), 
donde también son inadecuadas las condiciones de salubridad. Ello explica por qué las parasitosis intestinales son endémicas (Gámez A, comunicación personal, 1995).

Entre los problemas médicos producidos por las parasitosis intestinales destaca la anemia (3), cuya prevalencia es especialmente elevada en los países en desarrollo, donde oscila entre $20 \mathrm{y}$ $40 \%$ en mujeres no gestantes. Se ha observado que esta enfermedad, cuya principal causa en los países de la Región es la deficiencia de hierro, constituye un problema de salud muy difundido, especialmente entre mujeres embarazadas y niños. No obstante, las actividades de los programas nacionales de control son insuficientes y se han limitado a incluir suplementos de hierro en la dieta de las embarazadas que acuden a los servicios de control prenatal (1). Por otra parte, la anemia es la complicación más frecuente del embarazo y está asociada con tasas elevadas de parto pretérmino, bajo peso al nacer y mortalidad perinatal (4).

Entre las causas de anemia gestacional se encuentran la deficiencia de hierro; la deficiencia de ácido fólico, que produce anemia megaloblástica y se asocia con defectos del tubo neural; y, con menor frecuencia, la deficiencia de glucosa-6-fosfato deshidrogenasa, la drepanocitosis o anemia de células falciformes (5) y las talasemias (4). La anemia por deficiencia de hierro se produce generalmente por pérdida de sangre, ya sea como consecuencia de parasitosis intestinales, pérdidas menstruales o una mala alimentación (6).

Desde el punto de vista clínico, la mayor atención recae en la anemia del embarazo y por ello es frecuente observar en los servicios de ginecología y obstetricia la administración de suplementos de hierro a las embarazadas $(7,8)$. Esta medida está destinada a prevenir la anemia y sus consecuencias en el feto y recién nacido $(9,10)$.

Aunque no se conoce con exactitud la prevalencia de anemia y de deficiencia de hierro en las Américas, se calcula que en muchos países alrededor de $60 \%$ de las mujeres embarazadas padecen estos trastornos (11). Se sabe que la prevalencia de anemia varía de un país a otro y que depende de factores tales como la carga de malnutrición, malaria (12), parasitosis intestinales o drepanocitosis (5). Por citar algunas cifras, en Santiago de Chile la prevalencia de anemia en mujeres gestantes es de cerca de 1,2\% (13), mientras que en México se ha estimado que oscila alrededor de 18\% (14).

En el Perú, la anemia se ha estudiado en poblaciones situadas principalmente en la costa. Los estudios realizados sobre este tema en la selva peruana son escasos y no se dispone de estimaciones confiables de la prevalencia de anemia durante la gestación.

El presente estudio se realizó con objeto de estimar la prevalencia de anemia en mujeres embarazadas que acuden al servicio de ginecología y obstetricia del Hospital Regional de Pucallpa, localizado en la selva del Perú, y su posible asociación con la edad cronológica de la madre y con su grado de escolaridad, paridad, peso al inicio del embarazo y número de controles prenatales. También se intentó averiguar si la concentración de hemoglobina en la sangre de las embarazadas se asocia con el peso del recién nacido.

\section{MATERIALES Y MÉTODOS}

En este estudio, de diseño transversal, se utilizaron como fuentes de datos los registros de control prenatal o del parto del servicio de ginecología y obstetricia del Hospital Regional de Pucallpa. El período de la investigación se extendió de enero de 1993 a junio de 1995.

La muestra estudiada estuvo integrada por 1015 registros de control prenatal, correspondientes a una o más consultas realizadas a dicho servicio por mujeres embarazadas. El control prenatal se consideró adecuado cuando la embarazada acudió al servicio más de tres veces durante la gestación. Algunas embarazadas que recibieron atención prenatal dieron a luz en su casa, donde fueron atendidas por parteras tradicionales.
Los datos obtenidos en este estudio fueron los siguientes: edad de la madre, nivel de escolaridad, estado civil, número de veces que fue atendida en el servicio de atención prenatal, intervalo intergenésico (definido como el lapso en meses transcurrido entre un parto y el inicio del siguiente embarazo), paridad, peso al inicio y al final de la gestación, concentración de hemoglobina en la sangre (medida mediante el método de la cianmetahemoglobina en el tercer trimestre de la gestación), peso del recién nacido y número de hijos nacidos vivos y muertos. Asimismo, se calculó el aumento de peso durante el embarazo.

La anemia se definió como una concentración de hemoglobina menor de $11 \mathrm{~g} / \mathrm{dL}$, conforme a los criterios de la OMS (3), y su gravedad se estratificó del siguiente modo: grave $(<7 \mathrm{~g} / \mathrm{dL})$, moderada (de 7 a $9 \mathrm{~g} / \mathrm{dL}$ ) y leve $(>9$ a $<11 \mathrm{mg} / \mathrm{dL}$ ). Se consideraron normales las concentraciones de hemoglobina de $11 \mathrm{~g} / \mathrm{dL}$ o mayores. La muerte fetal se definió como el fallecimiento del feto a partir de la vigésima octava semana de gestación.

La prevalencia de anemia se estimó primero para todas las gestantes y posteriormente se estratificó en función de su edad, número de gestaciones previas, nivel de escolaridad, intervalo intergenésico y aumento de peso durante el embarazo. También se estimaron la prevalencia de muerte fetal y de recién nacidos de bajo peso.

Por otro lado, se investigó la relación entre la gravedad de la anemia y las medias aritméticas del peso del recién nacido, del aumento de peso de la gestante durante el embarazo y del peso al inicio de la gestación. Las diferencias entre las medias de estas variables se evaluaron por medio de análisis de la varianza de una vía, y entre pares de medias con la prueba de Scheffé. La homogeneidad de las varianzas se valoró utilizando la prueba de igualdad de varianzas de Bartlett. En el presente estudio, las varianzas de todas las muestras fueron homogéneas, por lo que no fue necesario transformar los datos. 
La relación entre el peso del recién nacido y la concentración de hemoglobina se estimó mediante el coeficiente de correlación parcial de Pearson. Por último, se construyó un modelo de regresión múltiple a fin de estimar las asociaciones entre el peso del recién nacido (variable dependiente del modelo) y las siguientes variables independientes: edad cronológica de la madre, nivel de escolaridad, número de controles prenatales, peso al inicio del embarazo, aumento de peso, paridad y concentración de hemoglobina.

\section{RESULTADOS}

La edad de las gestantes incluidas en este estudio estuvo comprendida entre los 13 y 46 años. De esta muestra, $24,1 \%$ (intervalo de confianza [IC] de 95\%: 17,0 a 31,1\%) eran menores de 19 años, $69,1 \%$ (IC: 64,6 a 73,5\%) tenían de 20 a 35 años y 6,77\% (IC: 0,8 a $14,8 \%$ ) eran mayores de 35 .

La prevalencia estimada de anemia entre las gestantes que acudieron al servicio de ginecología y obstetricia del Hospital Regional de Pucallpa fue de 70,3\% (IC: 65,8 a 74,8\%). La anemia leve fue la más frecuente $(67,7 \%$; IC: $62,2$ a $73,2 \%)$, seguida de la anemia moderada (27,9\%; IC: 19,6 a $36,2 \%)$ y grave $(4,4 \%$; IC: $-5,1$ a $13,9 \%)$. La prevalencia de anemia fue independiente de la edad materna. Así, en las menores de 19 años la prevalencia fue de $72,7 \%$ (IC: 65,2 a $80,1 \%$ ); en las gestantes que tenían de 20 a 35 años, de $69,1 \%$ (IC: 64,6 a $73,6 \%$ ); y en las mayores de 35 años, de 74,4\% (IC: 60,7 a $88,1 \%$ ) (ji cuadrado $\left[\chi^{2}\right]$ de Pearson $=$ $0,95 ; P=0,62)$. Como muestra el cuadro 1 , la prevalencia de anemia en general y especialmente la de anemia moderada aumentó con la paridad. En las mujeres que habían estado embarazadas un máximo de tres veces, la prevalencia ascendió a 69\% (IC: 63,9 a $73,4 \%$ ) y en las que habían tenido más de tres embarazos, a $74 \%$ (IC: 67,9 a $80,1 \%$ ) ( $\chi^{2}$ de Pearson 1,77; $P=0,183)$.

La prevalencia de anemia no varió según el nivel de escolaridad de las madres estudiadas (cuadro 2). Empero, fue mucho más alta en las anémicas que en aquellas cuyas concentraciones de hemoglobina eran normales.

La prevalencia de anemia tampoco varió según el intervalo intergenésico: para períodos menores de 24 meses fue de $73,2 \%$ (IC: 62,9 a $83,5 \%$ ); para períodos de 24 a 36 meses, de $62 \%$ (IC: $50,7$ a $73,3 \%)$, y para períodos mayores de 36 meses, de 72\% (IC: 65,2 a $78,8 \%)\left(\chi^{2}\right.$ de Pearson $=2,86 ; P=$
$0,239)$. En cambio, la prevalencia de anemia se asoció con el aumento de peso durante el embarazo. El cuadro 3 muestra que en las mujeres que aumentaron más de $12 \mathrm{~kg}$ de peso la prevalencia de anemia fue menor (58\%; IC: 43,2 a $71,8 \%$ ) que en las que aumentaron menos de $9 \mathrm{~kg}(80,4 \%$; IC: $71,8$ a $87,3 \%)$. En estas últimas, la prevalencia de anemia fue mayor que la prevalencia global en todas las mujeres estudiadas (70,3\%; IC: 65,8 a

CUADRO 1. Porcentaje de gestantes sin anemia y con anemia leve, moderada y grave, según la paridad. Servicio de Ginecología y Obstetricia, Hospital Regional de Pucallpa, Perú, 1993-1995

\begin{tabular}{|c|c|c|c|c|c|c|}
\hline \multirow[b]{3}{*}{ Anemia } & \multicolumn{4}{|c|}{ Partos previos } & \multirow[b]{3}{*}{$\chi^{2}$} & \multirow[b]{3}{*}{$P$} \\
\hline & \multicolumn{2}{|c|}{$\leq 3$} & \multicolumn{2}{|c|}{$>3$} & & \\
\hline & $\%$ & IC95\% & $\%$ & IC95\% & & \\
\hline Grave & 3,0 & 1,5 a 5,3 & 3,5 & 1,4 a 7,0 & 5,18 & 0,023 \\
\hline Moderada & 16,1 & 12,5 a 20,2 & 25,5 & 19,6 a 32,1 & 7,35 & 0,007 \\
\hline Leve & 49,6 & 44,4 a 54,8 & 45,0 & 38,0 a 52,2 & 1,09 & 0,296 \\
\hline Ninguna & 31,3 & 22,6 a 36,4 & 26,0 & 20,0 a 32,6 & 1,77 & 0,183 \\
\hline
\end{tabular}

$\chi^{2}$ (ji cuadrado de Pearson para el modelo general) $=7,85 ; P=0,049$.

IC95\% = intervalo de confianza de $95 \%$.

CUADRO 2. Presencia y ausencia de anemia (\%) según el nivel de escolaridad de las gestantes atendidas en el Hospital Regional de Pucallpa, Perú, 1993-1995

\begin{tabular}{lcccccc}
\hline \multicolumn{1}{c}{$\begin{array}{c}\text { Nivel de } \\
\text { escolaridad }\end{array}$} & $\begin{array}{c}\text { Gestantes } \\
\text { sin anemia }\end{array}$ & (IC95\%) & $\begin{array}{c}\text { Gestantes } \\
\text { con anemia }\end{array}$ & (IC95\%) & $\chi^{2}$ & $P$ \\
\hline Ninguno & 20,0 & $(-15,0$ a 55,0) & 80,0 & $(45,0$ a 115,0) & 0,23 & 0,63 \\
Primaria & 28,4 & $(19,3$ a 37,5) & 71,6 & $(62,5$ a 80,7) & 0,12 & 0,72 \\
Secundaria & 28,7 & $(24,4$ a 33,0) & 71,3 & $(67,0$ a 75,6) & 1,09 & 0,30 \\
Estudios superiores & 44,7 & $(30,5$ a 58,9) & 55,3 & $(41,1$ a 69,5) & 5,31 & 0,02 \\
\hline
\end{tabular}

$\chi^{2}$ (ji cuadrado de Pearson para el modelo general $)=5,50 ; P=0,139$. IC $95 \%$ = intervalo de confianza de $95 \%$.

CUADRO 3. Presencia o ausencia de anemia (\%) según el aumento de peso durante la gestación en embarazadas atendidas en el Hospital Regional de Pucallpa, Perú, 1993-1995

\begin{tabular}{ccccccc}
\hline $\begin{array}{c}\text { Aumento de } \\
\text { peso }(\mathrm{kg})\end{array}$ & $\begin{array}{c}\text { Gestantes } \\
\text { sin anemia }\end{array}$ & $($ IC95\%) & $\begin{array}{c}\text { Gestantes } \\
\text { con anemia }\end{array}$ & $($ IC95\%) & $\chi^{2}$ & $P$ \\
\hline$<9$ & 19,6 & $(12,7$ a 28,2$)$ & 80,4 & $(71,8$ a 87,3) & 7,94 & 0,005 \\
$9-12$ & 32,1 & $(20,3$ a 45,9) & 67,9 & $(54,0$ a 79,7$)$ & 0,64 & 0,421 \\
$>12$ & 42,0 & $(28,2$ a 56,8$)$ & 58,0 & $(43,2$ a 71,8$)$ & 6,32 & 0,012 \\
\hline
\end{tabular}

$\chi^{2}$ (ji cuadrado de Pearson para el modelo general $)=9,22 ; P=0,01$.

IC $95 \%$ = intervalo de confianza de $95 \%$. 
$74,8 \%$ ). De siete gestantes con anemia grave, seis (86\%; IC: 60,3 a 111,7\%) aumentaron menos de $9 \mathrm{~kg}$ durante el embarazo. En cambio, en las embarazadas que aumentaron más de $12 \mathrm{~kg}$ no se observó ningún caso de anemia grave $(0 \%$; IC: 0,0 a $7,1 \%)$ y entre ellas la anemia moderada fue menos frecuente que en las mujeres que aumentaron menos de peso. Asimismo, entre las mujeres que aumentaron más de $12 \mathrm{~kg}$, el porcentaje de las que tenían concentraciones de hemoglobina normales fue mayor (42\%; IC: 28,2 a $56,8 \%$ ) que el de mujeres con hemoglobina normal observado globalmente en las embarazadas atendidas en el Hospital de Pucallpa (29,5\%; IC: 25,7 a 33,3\%).

Si se adopta el postulado de que las gestantes que aumentaron más de $12 \mathrm{~kg}$ de peso durante el embarazo presentaban hemoconcentración y por lo tanto se excluyen del análisis, la prevalencia de anemia en las embarazadas asciende a 76,2\%, (IC: 69,7 a 82,7\%) y la ausencia de anemia se reduce a $23,8 \%$ (IC: 17,3 a 30,3\%). Con esta modificación, la prevalencia de anemia grave fue de $4,2 \%$ (IC: 1,2 a $7,2 \%$ ), la de anemia moderada, de 20,2\% (IC: $14,1$ a $26,3 \%)$, y la de anemia leve, de 51,8\% (IC: 44,2 a 59,4\%).

Mediante el análisis de la varianza de una vía se compararon los cuatro grupos con anemia grave, moderada y leve y sin anemia, y se encontró que cuanto menos peso aumentaron las gestantes durante el embarazo, mayor fue la gravedad de su anemia (cuadro 4). Hubo una diferencia significativa entre las madres sin anemia y las que tenían anemia de cualquier grado, pero no hubo ninguna diferencia en relación con el grado de la anemia. Por otra parte, en los grupos estudiados no se encontraron diferencias entre el peso de la madre al inicio del embarazo y la gravedad de su anemia gestacional. En las gestantes con anemia grave el aumento de peso fue de $59,03 \pm 3,89 \mathrm{~kg}$ (media \pm error estándar); en las que padecieron anemia moderada, 55,04 $\pm 1,17 \mathrm{~kg}$; en las que desarrollaron anemia leve, 53,78 \pm 0,72 $\mathrm{kg}$; $\mathrm{y}$ en las que no tuvieron anemia, $55,36 \pm 1,12 \mathrm{~kg}(F=1,20 ; P=0,30)$.
CUADRO 4. Aumento de peso (media en kg \pm error estándar) durante la gestación según la gravedad de la anemia, en embarazadas atendidas en el Hospital Regional de Pucallpa, Perú, 1993-1995

\begin{tabular}{lrc}
\hline & \multicolumn{2}{c}{ Aumento de peso } \\
\cline { 2 - 3 } Anemia & Media & Error estándar \\
\hline Grave & 5,68 & $(1,59)$ \\
Moderada & 8,46 & $(0,65)$ \\
Leve & 8,52 & $(0,40)$ \\
Ninguna & 10,45 & $(0,64)$ \\
\multicolumn{1}{c}{ Total } & 8,96 & $(0,31)$ \\
\hline
\end{tabular}

$F=4,11 ; P=0,0073$.

La supervivencia fetal se analizó en 557 gestaciones: 536 productos nacieron vivos y 19 fueron muertes fetales tardías. Solo se produjeron dos muertes fetales después del parto, lo cual elevó la mortalidad perinatal a 37,7 por 1000 gestaciones. Las muertes fetales no se asociaron con las concentraciones de hemoglobina en la sangre de la madre. En Pucallpa, 56,3\% de las 19 madres cuyos hijos nacieron muertos padecían anemia, y este porcentaje fue menor que la prevalencia de anemia estimada en todas las embarazadas de la muestra $(70,3 \%)$. Cuando se compararon las concentraciones de hemoglobina de las mujeres cuyos hijos nacieron muertos con las de todas las embarazadas, la proporción de anémicas fue similar, lo cual permite descartar la asociación entre anemia materna y mortalidad neonatal. Las causas de mortalidad más frecuentes fueron ruptura prematura de membranas, embarazo múltiple, desprendimiento prematuro de la placenta y anencefalia. Los dos casos de muerte posparto se debieron a malformaciones congénitas.

La media del peso de los recién nacidos en el Hospital de Pucallpa fue de 3092 g y no mostró ninguna asociación con la gravedad de la anemia. La media del peso de los recién nacidos de madres con anemia grave fue de $3095 \mathrm{~g}( \pm 184,11)$, la de los hijos de madres con anemia moderada, 3014,86 g $( \pm 70,28)$, la de los hijos de madres con anemia leve, $3092,99 \mathrm{~g}$ $( \pm 37,39)$, y la de los hijos de madres sin anemia, $3137,74 \mathrm{~g}( \pm 50,20)$. Según los resultados del análisis de regresión, el peso del recién nacido tampoco se asoció con la concentración de hemoglobina $\left(R^{2}=0,0082 ; P=\mathrm{NS}\right)$.

De los niños que nacieron en el Hospital de Pucallpa durante el período abarcado por el estudio, 56 presentaron bajo peso al nacer, lo cual equivale a una proporción de este trastorno igual a 10,4\% (IC: 7,8 a 13,0\%). La media de la concentración de hemoglobina de las madres de estos niños fue de 9,87 g/dL $( \pm 0,25)$, cifra similar a la de toda la población de gestantes atendidas en el hospital durante el mismo período $(10,03 \mathrm{~g} / \mathrm{dL})( \pm 0,07)$. La proporción de recién nacidos de bajo peso entre madres que padecían anemia fue de 13\% (IC: 9,0 a 17,0\%) y entre madres que no la padecían (IC: $6,2$ a 18,2$)$, de $12 \%$.

Según los resultados del modelo de regresión múltiple, el peso de la madre al inicio de la gestación, su aumento de peso durante la misma y la paridad se asociaron con el peso del recién nacido y estas tres asociaciones ajustadas fueron directas (cuadro 5). Por otra parte, en las mujeres que se sometieron a un control prenatal adecuado, la concentración de hemoglobina fue mayor $(10,16 \mathrm{~g} / \mathrm{dL} ; \pm 0,08)$ que en las que no recibieron ningún control $(9,78$ $\mathrm{g} / \mathrm{dL} ; \pm 0,13)(F=6,73 ; P=0,0097)$.

\section{DISCUSIÓN}

La prevalencia de anemia ferropénica ha descendido notablemente en los países desarrollados (15), aunque la prevalencia mundial no ha cambiado. Se estima que este tipo de anemia afecta a más de 500 millones de personas en todo el mundo (15).

La anemia ferropénica tiene consecuencias importantes para la salud pública, ya que causa trastornos del desarrollo psicomotor y de las funciones cognoscitivas en preescolares, reduce el rendimiento laboral de los adultos, y aumenta la frecuencia de bajo peso al nacer, de parto prematuro y de mortalidad perinatal (15). En los países meridionales se ha observado que la deficiencia de hierro se asocia con una reducción de la productividad (15). 
CUADRO 5. Resultados del modelo de regresión múltiple aplicado al peso del recién nacido y a distintas variables maternas. Hospital Regional de Pucallpa, Perú, 1993-1995

\begin{tabular}{lcc}
\hline \multicolumn{1}{c}{ Variable materna } & $\begin{array}{c}\text { Coeficiente } \\
\text { (error estándar) }\end{array}$ & IC95\% \\
\hline Constante & $951,22(20,28)$ & 9,84 a 1482,59 \\
Edad (años) & $-7,89(5,69)$ & $-19,07$ a 3,28 \\
Analfabetismo & $-45,58(344,29)$ & 721,95 a 630,79 \\
Escuela primaria & $55,95(97,02)$ & $-134,66$ a 246,56 \\
Escuela secundaria & $59,34(82,69)$ & $-103,10$ a 221,78 \\
Estado civil & $-16,10(80,66)$ & $-174,57$ a 142,36 \\
Control prenatal & $10,18(43,55)$ & $-75,38$ a 95,74 \\
Intervalo intergenésico & & \\
$\quad<24$ meses & & $-180,06$ a 97,57 \\
$\quad 24-36$ meses & $-41,24(70,66)$ & $-74,89$ a 203,13 \\
Peso inicial & $64,12(70,76)$ & 23,48 a 38,04 \\
Aumento de peso & $30,76(3,71)$ & 31,28 a 62,52 \\
Paridad & $46,90(7,95)$ & 10,36 a 67,84 \\
Concentración de hemoglobina & $39,10(14,63)$ & $-25,70$ a 37,69 \\
\hline
\end{tabular}

IC95\% = intervalo de confianza de $95 \%$.

$R^{2}=0,195$.

$P=0,00001$

La prevalencia de anemia en las gestantes comprendidas en este estudio (70,3\%; IC: 65,8 a $74,8 \%$ ) fue mayor que la estimada en las embarazadas de toda la Región (60\%) (11). En los países del Caribe de habla inglesa, la prevalencia de anemia en mujeres embarazadas difiere de un país a otro y, según diversos estudios, oscila entre 27 y $70 \%(16$, 17). Por ejemplo, en la isla de Granada se estimó una prevalencia de $63 \%$ (18) y en Jamaica, de $52 \%$ (18). En otro estudio realizado en las Islas Turcas y Caicos y publicado en 1989 se notificó que, si bien la prevalencia de anemia era alta (60-70\%), el porcentaje de gestantes con concentraciones de hemoglobina menores de $9 \mathrm{~g} / \mathrm{dL}$ se encontraba entre $7 \mathrm{y}$ $9 \%$ (16), estimación mucho menor que la descrita en el presente estudio.

En la Región ha habido pocas investigaciones sobre la anemia en embarazadas y entre ellas destacan dos estudios efectuados en el Brasil. En el primero, la prevalencia en primigestas de 9 a 15 años de edad fue de $8,5 \%$ (19) y en el segundo, que se llevó a cabo en parturientas de Porto Alegre, se encontró una prevalencia de 13,7\% (20). En estudios efectuados en Chile, México y Bolivia, las prevalencias estimadas fueron, respectivamente, $1,2 \%$ (13), $18,2 \%$ (14) y $25 \%$. De las mujeres anémicas detectadas en este último país, $19 \%$ tenían concentraciones de hemoglobina entre 8 y $11 \mathrm{~g} / \mathrm{dL}$ y $6 \%$ tenían concentraciones menores de 8 $\mathrm{g} / \mathrm{dL}$ (21), cifras menores que las obtenidas en la presente investigación.

En el Brasil se ha observado que una causa de anemia en el embarazo es la desnutrición por parasitosis (3). Dado que el estudio realizado en el Hospital Regional de Pucallpa se basa en un diseño transversal y en datos obtenidos de registros, no es posible establecer las causas de anemia más frecuentes entre las gestantes que acudieron a dicho hospital. No obstante, es posible que las parasitosis entéricas figuren entre las causas principales de las anemias diagnosticadas, sobre todo si se tiene en cuenta que la prevalencia de estas parasitosis en la zona asciende a casi 80\% (Gámez A, comunicación personal, 1995).

En este estudio no se ha observado que la prevalencia de anemia se modifique por efecto de la edad materna, el nivel de escolaridad, el intervalo intergenésico, ni el peso de la embarazada al inicio de la gestación. En cambio, dicha prevalencia sí varió en función de la paridad y del aumento de peso de la embarazada durante la gestación. El aumento de la prevalencia relacionado con la paridad podría estar asociado con el agotamiento de las reser- vas nutritivas de la embarazada (22), hipótesis que se ha postulado en otros estudios (22-24). Diallo et al. (24) encuentran que las primíparas y las grandes multíparas corren un riesgo más alto de anemia que otras embarazadas.

El peso de las mujeres estudiadas al inicio de la gestación puede considerarse adecuado (25). El peso al inicio del embarazo de las gestantes que fueron estudiadas en una zona rural de Costa Rica fue ligeramente menor que el encontrado en Pucallpa y tuvo un promedio que osciló alrededor de 52,1 $\mathrm{kg}$ (26). El mayor peso observado en las gestantes de Pucallpa podría relacionarse con su pertenencia a una zona urbana, en contraste con los datos de Costa Rica, que proceden de una zona rural.

El aumento de peso promedio durante la gestación fue de $8,96 \mathrm{~kg}$ en las embarazadas de Pucallpa y de $7,9 \mathrm{~kg}$ en las del estudio costarricense (26), y el porcentaje de recién nacidos de bajo peso en estos estudios fue de 10,4 y $9,2 \%$, respectivamente. Aunque el peso al nacer suele reducirse cuando es pobre la ingesta energética de la embarazada, en el estudio realizado en Costa Rica se observó que la ingesta de menos de $1000 \mathrm{kcal} /$ día se asocia con un peso al nacer dentro de lo normal (media cercana a $2900 \mathrm{~g}$ ) (26). Es probable que se precisen reducciones muy notables de la ingesta calórica durante el embarazo para que aumente sustancialmente la proporción de recién nacidos de bajo peso.

En una investigación llevada a cabo en la ciudad de Guatemala (27), la infestación de las embarazadas por helmintos se asoció con un retraso del crecimiento intrauterino. Dicha asociación persistió aun después de ajustar los datos según variables socioeconómicas y el nivel nutricional de la madre. En dicho estudio se concluyó que hasta $10 \%$ de los casos de retraso del crecimiento intrauterino pueden atribuirse a la infestación parasitaria de madres desnutridas. En el presente estudio, que se llevó a cabo en una zona donde la parasitosis intestinal es endémica y donde es alta la prevalencia de anemia, se ha estimado una proporción de recién nacidos de bajo peso 
de 10,4\%, valor inferior al estimado en 1980 en países en desarrollo (18\%) y más alto que el 7\% de los Estados Unidos en $1996(28,29)$.

En la actualidad se sigue debatiendo si la anemia del embarazo ejerce algún efecto en el peso del recién nacido. En un estudio realizado en el Brasil no se encontró asociación alguna entre estas dos variables (30), mientras que en otra investigación en ese mismo país se observó que la proporción de recién nacidos de bajo peso fue más alta entre gestantes anémicas que acudieron a la primera consulta prenatal en el tercer trimestre del embarazo. En distintas revisiones sobre anemia y embarazo, esta última se ha asociado con bajo peso al nacer $(4,10,14,15,31-33)$. Aunque en el presente trabajo se estimó una alta prevalencia de anemia gestacional, no se detectó ninguna asociación con bajo peso al nacer. El análisis multivariante reveló que el peso de la madre al inicio del embarazo, el aumento de peso durante la gestación y la paridad están directamente asociados con el peso del recién nacido. A diferencia de otros estudios en que se detectó una asociación entre anemia materna y peso al nacer, la presente investigación reveló que en Pucallpa el peso promedio de la madre al inicio de la gestación era mayor de $50 \mathrm{~kg}$ $(54,6 \mathrm{~kg})$ y por lo tanto adecuado (15, 34), aun entre embarazadas anémicas.

Varios investigadores han notificado un peso promedio significativamente mayor en los hijos de madres no anémicas que en los hijos de madres con anemia, y una prevalencia de neonatos de bajo peso significativamente más alta entre madres anémicas, sin que hubiera asociación entre la concentración de hemoglobina de la madre y el peso del recién nacido (32). En el presente estudio tampoco se encontró ninguna asociación entre estas dos últimas variables. Asimismo, la media del peso de los recién nacidos de madres anémicas fue similar a la de los hijos de madres no anémicas. Por otra parte, la prevalencia de hijos de bajo peso entre madres anémicas fue similar a la estimada entre madres sin anemia. De ahí que diversas variables, tales como el control prenatal, puedan ser factores que confunden la asociación entre el peso del recién nacido y la presencia o ausencia de anemia en la madre. Es probable que la deficiencia de hierro de la madre no se manifieste en el feto. La fisiología de la eritropoyesis materna y fetal durante el embarazo muestra que su estimulación se produce de forma independiente en la circulación materna y fetal. La eritropoyetina es el principal factor regulador de la hemopoyesis en ambos sistemas circulatorios y la placenta constituye una barrera que evita el paso de eritropoyetina de la madre al feto (35). Esto indica que la concentración de hemoglobina en la madre y el feto no son necesariamente dependientes entre sí. Acaso ello explique por qué la concentración de hemoglobina en la sangre materna no muestra asociación alguna con su concentración en la sangre del cordón umbilical (36).

Si bien el peso al inicio del embarazo fue similar en las mujeres con y sin anemia, el aumento de peso durante la gestación varió en ambos grupos. La prevalencia de anemia fue mayor en las mujeres cuyo aumento ponderal fue menor. Cabe señalar, sin embargo, que un aumento de peso mayor de 12 $\mathrm{kg}$ no es normal y puede asociarse con preeclampsia. En esta situación se produce hemoconcentración, la cual eleva la concentración de hemoglobina y por lo tanto puede enmascarar una anemia verdadera (37). La anemia gestacional también se ha asociado con una mayor tasa de mortalidad perinatal en diversos estudios $(24,38)$.

Aunque la deficiencia de hierro se ha considerado un problema grave de salud pública, su tratamiento ha suscitado polémicas, ya que algunos investigadores han notificado que las mujeres con anemia ferropénica son muy susceptibles a las infecciones, en tanto que otros han observado que las infecciones son más frecuentes en las que tienen sobrecargas de hierro. Numerosos microorganismos precisan hierro para crecer y desarrollarse. El aumento de la concentración sérica de transferrina no saturada durante una infección reduce al mínimo la disponibilidad de hierro para dichos microorganismos y, por ello, el tratamiento específico de la anemia ferropénica en pacientes con infecciones podría ser contraproducente si no se administra de modo racional (39).

Si bien este estudio no demuestra que exista una asociación entre la anemia materna y el peso del recién nacido, cabe señalar que la anemia se ha asociado con una producción insuficiente de leche materna que puede reducir la duración de la lactancia materna exclusiva (40). Del mismo modo, los hijos de madres anémicas tienden a padecer anemia cuando la causa de la misma es la deficiencia de hierro.

El peso del recién nacido puede considerarse un importante factor predictor de su supervivencia y de su desarrollo mental y físico. En este estudio se ha observado que el peso de la madre al inicio del embarazo, el aumento de peso durante la gestación y la paridad son variables que se asocian directamente con el peso del niño al nacer. No obstante, en el análisis multivariante no se encontró ninguna asociación entre la concentración de hemoglobina materna y el peso del recién nacido. Otros investigadores han notificado que la paridad y el peso de la madre al inicio de la gestación se asocian inversamente con la proporción de hijos que nacen con bajo peso (33).

En síntesis, en este estudio se ha estimado que la prevalencia de anemia en las gestantes atendidas en el Hospital Regional de Pucallpa entre 1993 y 1995 es elevada, pero que la anemia materna no se asocia con bajo peso al nacer ni con la mortalidad perinatal. Los estudios que se realicen en el futuro deberían destinarse a investigar las principales causas de anemia en las gestantes de Pucallpa y sus efectos en el desarrollo psicomotor temprano de sus hijos.

Agradecimiento. Este estudio fue parcialmente apoyado por el LID Grant del Programa de Reproducción Humana de la OMS y por el Hospital Regional de Pucallpa. Los autores agradecen a los doctores Ángel Gutiérrez, Luis Makiya, Juan Taboada, Manuel Salazar y Ricardo Conde toda la ayuda prestada. 


\section{REFERENCIAS}

1. Gueri M. Deficiencias de micronutrientes en las Américas. Bol Oficina Sanit Panam 1994; 117:477-482.

2. Perú, Instituto Nacional de Estadística e Informática (INEI). Encuesta Demográfica y de Salud Familiar 1991/1992. Lima: INEI, PRISMA y Macrointernational Inc, eds.; 1992.

3. Petraglia B, Silva L, Chaves Netto H, Peixoto MA, Montenegro C. Anemia e gravidez. J Bras Ginecol 1990;100:51-53.

4. Lops VR, Hunter LP, Dixon LR. Anemia in pregnancy. Am Fam Physician 1995;51: 1189-1197.

5. Balgir RS. Age at menarche and first conception in sickle cell hemoglobinopathy. Indian Pediatr 1994;31:827-832.

6. Herrmann RP. Iron deficiency: current trends and fads. Aust Fam Physician 1994;23: 1457-1461.

7. Menéndez C, Todd J, Alonso PL, Francis N, Lulat $S$, Ceesay $S$, et al. The response to iron supplementation of pregnant women with haemoglobin genotype AA or AS. Trans $R$ Soc Trop Med Hyg 1995;89:289-292.

8. Buglanov AA, Saiapina Ev, Turaev AT. A comparative study of the effectiveness of iron preparations in the treatment of iron deficiency anemias in pregnant women. Akush Ginekol Mosk 1994;6:16-18.

9. Gadowski SL, Gale K, Wolfe SA, Jory J, Gibson R, O'Connor DL. Biochemical folate, B12, and iron status of a group of pregnant adolescents accessed through the public health system in southern Ontario. J Adolesc Health 1995; 16:465-474.

10. Boult BE, Cunningham PW. Some aspects of obstetrics in black teenage pregnancy: a comparative analysis. Med Law 1995;14:93-97.

11. Organización Panamericana de la Salud. Vol I, Las condiciones de salud en las Américas. Washington, DC: OPS;1994:257-258.

12. Matteelli A, Donato F, Shein A, Muchi JA, Leopardi O, Astori L, et al. Malaria and anemia in pregnant women in urban Zanzibar, Tanzania. Ann Trop Med Parasitol 1994;88: 475-483.

13. Hertrampf E, Olivares M, Letelier A, Castillo C. Iron nutritional status in pregnant adolescents at the beginning of gestation. Rev Med Chile 1994;122:1372-1377.

14. Martínez H, González-Cossio T, Flores M, Rivera-Dommarco J, Lezana MA, SepúlvedaAmor J. Anemia in women of reproduc- tive age: the results of a national probability survey. Salud Publica Mex 1995;37: 108-119.

15. Cook JD, Skikne BS, Baynes RD. Iron deficiency: the global perspective. Adv Exp Med Biol 1994;356:219-228.

16. Simmons WK, Been $\mathrm{H}$, Gallagher P, Patterson AW. Anemia in antenatals in the Turks and Caicos Islands. West Indian Med J 1987;36: 210-215.

17. Simmons WK, Wynter $\mathrm{H}$, Gallagher $\mathrm{P}$, Viteri F, Patterson AW. Hemoglobin levels in West Indian antenatals. West Indian Med I 1987; 36:216-224.

18. Simmons WK. Control de la deficiencia de hierro en el Caribe de habla inglesa. Bol Oficina Sanit Panan 1994;117:538-546.

19. Mathias L, Nestarez JE, Kanas M, Neme B. Gravidez na adolescencia: I, Primigestas de 9 a 15 anos. J Bras Ginecol 1985;95:89-91.

20. Giugliani ER, Jorge SM, Sopes-Gonçalves A. Folate and vitamin B12 deficiency among parturients from Porto Alegre, Brazil. Rev Invest Clin 1984;36:133-136.

21. Cardozo L, Zuna H, Urjel R, Saavedra F. Estudio de las causas de anemia en embarazadas de la Maternidad de Percy Boland de la ciudad de Santa Cruz, 1985. Bol Cient CENETROP 1985;11:58-67.

22. Villar J, Rivera J. Nutritional supplementation during two consecutive pregnancies and the interim lactation period: effect on birth weight. Pediatrics 1988;81;51-57.

23. Brunvand L, Henriksen C, Larsson M, Sandberg AS. Iron deficiency among pregnant Pakistanis in Norway and the content of phytic acid in their diet. Acta Obstet Gynecol Scand 1995;74:520-525.

24. Diallo MS, Diallo TS, Diallo FB, Diallo $Y$, Camara AY, Onivogui G, et al. Anemia and pregnancy: epidemiologic, clinical and prognostic study at the university clinic of the Ignace Deen Hospital, Conakry (Guinée). Rev Fr Gynecol Obstet 1995;90:138-141.

25. Omar MM, Hogberg U, Bergstrom B. Maternal health and child survival in relation to socioeconomic factors. Gynecol Obstet Invest 1994;38:107-112.

26. Murillo S, Ulate E, Mata L. Nutrición materna durante el embarazo: estudio de mujeres de una zona rural de Costa Rica. Bol Oficina Sanit Panam 1988;104:345-354.

27. Villar J, Klebanoff MA, Kestler E. The effect on fetal growth of protozoan and helminthic infection during pregnancy. Obstet Gynecol 1989;74:915-920.

28. World Health Organization. The incidence of low birth weight: a critical review of available information. World Health Stat 1980;33:197-224.

29. Guyer B, Martin JA, MacDorman MF, Anderson RN, Strobino DM. Annual summary of vital statistics-1996. Pediatrics 1997;100: 905-918.

30. Amar I, Soares MMO, Crespo GMS. Anemia carencial e peso e vitalidade do concepto. Arq Bras Med 1988;62:451-454

31. Nishimura A, Sakagushi A, Rozas A, Novo JLVG, Paiva ER, Neme B. Anemia materna e baixo peso fetal. Ginecol Obst Bras 1988;11: 110-114.

32. Swain S, Singh S, Bhatia BD, Pandey S, Krishna M. Maternal hemoglobin and serum albumin and fetal growth. Indian Pediatr 1997;31:777-782.

33. Hirve SS, Ganatra BR. Determinants of low birth weight: a community based prospective cohort study. Indian Pediatr 1994;31:1221-1225.

34. Spinillo A, Capuzzo E, Piazzi G, Nicola S, Colonna S, Iasci A. Maternal high risk factors and severity of growth deficit in small for gestational age infants. Early Hum Dev 1994; 38:35-43.

35. Huch R, Huch A. Erythropoietin in obstetrics. Hematol Oncol Clin North Am 1994;8:1021-1040.

36. Altinkaynak S, Alp H, Bastem A, Selimoglu $M$, Energin M. Serum ferritin and hemoglobin levels of mothers and their newborns. Turk J Pediatr 1994;36:289-293.

37. Yang JM, Wang KG. Relationship between acute fetal distress and maternal-placentalfetal circulations in severe pre-eclampsia. Acta Obstet Gynecol Scand 1995;74:419-424.

38. Lau TK, Li CY. A perinatal audit of stillbirths in a teaching hospital in Hong Kong. Aust NA J Obstet Gynecol 1994;51:214-220.

39. Álvarez Amaya C. Algunas consideraciones en relación con la administración de hierro. Bol Med Hosp Infant Mex 1994;51:214-220.

40. Henly SJ, Anderson CM, Avery MD, HillsBonczyk SG, Potter S, Duckett LJ. Anemia and insufficient milk in first time mothers. Birth 1995;22:86-92.

Manuscrito recibido el 30 de abril de 1996 y aceptado para publicación en versión revisada el 17 de abril de 1997 
ABSTRACT Population based health surveys in Peru show that the general fertility rates, proportion of pregnant adolescents, and maternal and child morbidity are higher in the jungle regions than in other parts of the country. Endemic intestinal parasitic diseases Prevalence of Anemia in
Pregnant Women, Pucallpa
Regional Hospital, Peru increase the risk of anemia in pregnant women already suffering from iron, folic acid, and other nutritional deficiencies. This is the most common complication of pregnancy in many Latin American countries and is often associated with premature labor, low birthweight, and perinatal mortality. There are very few studies on this subject based on jungle populations and no reliable estimates of the prevalence of anemia in local pregnant women. The present study was designed to determine the prevalence of anemia in pregnant women attending the Regional Hospital in Pucallpa, located in the Peruvian jungle, from January 1993 to June 1995. This crosssectional study, which was based on the registries of prenatal and childbirth services encompassing 1015 pregnant women, looked into the potential association between anemia and such variables as the mother's chronological age, schooling, previous pregnancies, and weight at the beginning of pregnancy. Maternal hemoglobin levels were compared with the newborns' weight at birth.

The prevalence of anemia in this population sample was $70.1 \%$. This value was not modified when adjusted for maternal age, schooling, or interval between births. Anemia prevalence was directly related to the number of previous pregnancies and inversely related to weight gain during pregnancy. The perinatal mortality rate was 37.7 per 1000 births. Neither this rate nor the birthweights were associated with the mother's degree of anemia. A multivariate regression analysis showed that maternal body weight at the start of pregnancy $(P=0.0001)$, weight gain during pregnancy $(P=0.0001)$, and the number of pregnancies $(P=0.008)$ are predictors of birthweight. Results showed that the high prevalence of anemia in Pucallpa's pregnant women is not associated with low birthweight or a high perinatal mortality rate. Future studies should investigate the principal causes of anemia in the pregnant women of Pucallpa and how the disease affects the psychomotor development of their offspring. 